\title{
Hematopoietic stem cells and the endosteal niche
}

\author{
Toshio Suda ${ }^{1}$ \\ ${ }^{I}$ Keio University, School of Medicine, Japan
}

Interaction of HSCs with their particular microenvironments, known as stem cell niches, is critical for maintaining the stem cell properties, including self-renewal capacity and the ability of differentiation into multiple lineages. Bonelining osteoblastic cells (OBs) and perivascular cells have been identified as major components of HSC niches. They express cell adhesion molecules and produce paracrine factors such as Angiopoietin-1 and thrombopoietin. In this presentation, I will show the role of the endosteal niche complex for the sustaining of quiescence of HSCs, and discuss the niche regulation through reactive oxygen species (ROS).

Cell Research (2008) 18:s90. doi: 10.1038/cr.2008.180; published online 4 August 2008

Correspondence: Toshio Suda

Toshio Suda, MD. Professor of Developmental Biology at The Sakaguchi Laboratory, School of Medicine, Keio University. Dr. Suda received MD from Yokohama City University School of Medicine, did his resident training at the Kanagawa Children's Medical Centre, and then as a research associate at Medical University of South Carolina. He became an assistant and associate professor at Jichi Medical School, and late a professor Kumamoto University School of Medicine and the Keio University. Dr Suda's major contributions include: 1) proposed together with Dr Makio Ogawa a stochastic model in the differentiation of stem cells 2) a pioneer in developing the purification methods for hematopoietic stem cells iKSL cells jand progenitors, 3) a pioneer in identification of the osteoblastic niche for the hematopoietic stem cells, and 4) established the relationship between oxidative stress and stem cell aging. Dr Suda is the current Director of Center for Integrated Medical Research Keio University, advisory Council RIKEN Kobe Institute, President (since 2003) Japanese Society of Stem Cell Research, and President (2008) of Japanese Society of Hematology. 